\title{
Mutations in Sodium Channel Gene SCN9A and the Pain Perception Disorders
}

\author{
Danica Marković, ${ }^{1}$ Radmilo Janković, ${ }^{1,2}$ and Ines Veselinović ${ }^{1}$ \\ ${ }^{1}$ Center for Anesthesiology and Reanimatology, Clinical Center Niš, Bulevar Dr. Zorana Djindjića 48, 18000 Niš, Serbia \\ ${ }^{2}$ Department for Anesthesia and Intensive Care, School of Medicine, University of Niš, Bulevar Dr. Zorana Djindjića 81, \\ 18000 Niš, Serbia
}

Correspondence should be addressed to Danica Marković; danica-amm@medfak.ni.ac.rs

Received 29 September 2014; Accepted 8 February 2015

Academic Editor: Sorin J. Brull

Copyright (C) 2015 Danica Marković et al. This is an open access article distributed under the Creative Commons Attribution License, which permits unrestricted use, distribution, and reproduction in any medium, provided the original work is properly cited.

\begin{abstract}
Voltage-gated sodium channels $(\mathrm{NaV})$ play a crucial role in development and propagation of action potentials in neurons and muscle cells. NaV1.7 channels take a special place in modern science since it is believed that they contribute to nerve hyperexcitability. Mutations of the gene SCN9A, which codes the $\alpha$ subunit of NaV1.7 channels, are associated with pain perception disorders (primary erythermalgia, congenital analgesia, and paroxysmal pain disorder). It is considered that the SCN9A gene mutations may cause variations in sensitivity to pain, from complete insensitivity to extreme sensitivity. Further research of the SCN9A gene polymorphism influence on pain sensitivity is essential for the understanding of the pathophysiology of pain and the development of the appropriate targeted pain treatment.
\end{abstract}

\section{Introduction}

Pain is the unpleasant physical or emotional feeling which occurs due to current or threatening tissue damage. Surgical stimulation is the most common predictable cause of pain and the duty of anesthesiologist is to provide an adequate amount of appropriate anesthetics in order to alleviate pain. Researches indicate that mild to extreme sensation of pain occurs in $30-70 \%$ of patients in postoperative period, which indicates that the pain treatment is not yet adequately developed. It is necessary to develop targeted pain therapy in order to relieve the postoperative recovery and reduce the total number of hospital days. Genetic researches enable the discovery of mutations which cause pain disorders. Diseases like primary erythromelalgia, paroxysmal pain disorder, and congenital analgesia may serve as adequate research models (Table 1). The causes of different pain perception in the general population may be investigated on these disease models. Targeting of the genes responsible for excessive perception of pain will enable the development of the targeted pain therapy and accurately determine the proper dosage [1].

\section{Sodium Channel Structure and Function}

Voltage-gated sodium channels $(\mathrm{NaV})$ play a key role in occurrence and propagation of action potentials in neurons and muscle cells. They allow the increase of sodium permeability through the cell membrane during the depolarisation phase. Nine isoforms of $\mathrm{NaV}$ channels differ in amino acid sequences and in a variety of physiological and pharmacological properties. Their activation has three states: deactivated (closed), activated (opened), and inactivated (closed) [1, 2].

$\mathrm{NaV}$ channels are large complexes of glycoproteins responsible for the initial phase of action potential in excitable cells. They consist of one $\alpha$ subunit and one or more $\beta$ subunits. Subunit $\alpha$ is a large transmembrane protein of approximately $260 \mathrm{kDa}$ with about 2000 amino acid residues. It is built out of four homologous domains (DI-DIV) which contain specific voltage sensors. Each homologous domain is built out of six transmembrane segments (S1-S6). Four domains conjoin inside the membrane and form a pore permeable for sodium. Sodium passes through these pores down the concentration gradient during the propagation of 
TABLE 1: Pain disorders and their characteristics.

\begin{tabular}{lccc}
\hline Disorder & IEM & PEPD & CIP \\
\hline Inheritance & AD & AD & AR \\
\hline Channel function & Increased & Increased & Absent \\
\hline Symptoms & $\begin{array}{c}\text { Painful feet and hands (burning) as the } \\
\text { response to heat and movement }\end{array}$ & $\begin{array}{c}\text { Burning in rectal, ocular, and mandibular } \\
\text { region, skin redness }\end{array}$ & No pain feeling at all \\
\hline \multirow{2}{*}{ Most frequent SNPs } & F1449V, L858F, L858H A863P, S241T, & M1627K & S459X, I767X, W897X, \\
& I848T, I234T, and F216S & M899I, and M932L \\
\hline
\end{tabular}

action potential. Every $\alpha$ subunit is associated with one or more $\beta$ subunits $(\beta 1-\beta 4)$ which represent transmembrane proteins with one extracellular IgG loop and one intracellular $\mathrm{C}$ end. This connection affects the total number of these channels on the cell surface, the sensitivity to voltage changes, the kinetics of $\alpha$ subunits, and the connections with other signaling molecules and cytoskeleton molecules. Mutations of the genes which code the $\alpha$ subunit created nine genes in total during the evolution in mammals. These genes code active channels and differ in tissue specificity and biophysical properties. Mutation of these genes causes substitution of a single amino acid and is associated with various diseases. Some of them are pain sensitivity disorders, epilepsy, various types of paralysis, ataxia, arrhythmia, muscle tissue diseases, tremor, dystonia, and so forth. It is considered that they are also responsible for some psychiatric diseases [3-6].

\section{NaV1.7 Channels}

Channels NaV1.7 are also called the voltage-gated sodium channel type IV $\alpha$ subunit. They are coded by the SCN9A gene and are highly expressed in sensory neurons of the dorsal roots, trigeminal ganglion, and sympathetic neurons ganglia, mainly in the nociceptors [2]. They are built out of 1977 amino acids (approximately $225 \mathrm{kDa}$ ) [6].

Immunohistochemical studies have shown that NaV1.7 is also present on the distal neurite ends, close to the impulse trigger zone. These channels show the possibility of depolarization even on minor stimuli and their biophysical function is to conduct stimuli which are below threshold. They are characterized by slow inactivation and slow recovery from inactivation. This allows them to answer to small depolarization stimuli and to amplify generation potentials on nerve endings. Scientists believe that the role of NaV1.7 is to contribute to nerve hyperexcitability $[2,6-8]$.

\section{The Genetic Basis of NaV1.7}

SCN9A gene is coding the $\alpha$ subunit of NaV1.7 and is primarily expressed in neurons of the dorsal root ganglia, sympathetic neurons ganglia, Swann cells, and neuroendocrine cells [9]. It is located on the 2 q24.3 chromosome and consists of 26 exons $[6,10]$.

Subunits $\alpha$ are coded by the SCN1A-SCN5A and SCN8ASCN11A genes, while subunits $\beta$ are coded by the SCN1BSCN4B genes [6]. Channel activity and function are regulated depending on which $\beta$ subunit is associated with the $\alpha$ domain [3-5]. Researchers have shown that mutations in the gene SCN9A are also associated with diseases whose base lies in the disorders of pain perception. This includes primary erythermalgia, congenital analgesia, and paroxysmal pain disorder. According to this, scientists have concluded that the SCN9A gene mutation can cause variations in the sensitivity to pain from complete insensitivity to extreme sensitivity [9].

\section{Primary Erythermalgia and SCN9A Gene Mutation}

Primary (inherited) erythromelalgia (IEM) is an autosomal dominant disease and represents a rare neurovascular disorder. It is characterized by the appearance of extreme neuropathic pain in the form of burning in the extremities as the response to heat or movement. Most patients feel the first symptoms in the early childhood (5-6 years); however, in some families symptoms can appear not earlier than in the old age. It is considered that the onset of symptoms depends on gene splicing as well as mutual combination of exons [11].

This disorder is highly resistant to therapy. Cooling of extremities relieves pain in the early stages; however, the later stages of the disease require the use of anesthetics [11]. Two reports describe the partial relief of pain in the patients with IEM using lidocaine and mexiletine $[12,13]$. The other study conducted by Legroux-Crespel et al. describes the relief of pain in the patients with IEM with oral administration of mexiletine $[14,15]$. It has been shown that it comes to the resistance to the anesthetic infusions after a while in some patients, as well as the resistance to the anesthetic creams (mexiletine, fentanyl, and lidocaine) [11]. Davis and Sandoroni have shown that in the case of patients with IEM, lidocaine relieves pain in only $55 \%$ of cases [13]. It is well known that in the basis of IEM lies a genetic mutation. However, there are numerous mutations which have to be considered and a great number of researches have been conducted with the aim of detecting the specific cause as well as finding a targeted and effective disease treatment [16].

The genetic basis of IEM is found on the second chromosome as it was observed as the existence of recombinations in the genetic region between D2S2370 and D2S1776, which can be found on 2q31-32. Yang et al. have revealed the connection between this syndrome and the $5.98 \mathrm{cM}$ region on the chromosome 2q24.2-q24.3. Further sequencing on 
the selected candidate genes has defined the first missense mutations on the SCN9A gene (I848T, L858F) [1, 6].

To date, 19 mutations associated with IEM have been found, including 18 missense mutations which cause substitution of one amino acid and one in-frame deletion. The penetration of IEM mutations is almost $100 \%$ and they all move the activation of NaV1.7 in the direction of hyperpolarization and slow deactivation $[6,17]$. The most important mutations in current genetic research are F1449V, L858F, L858H A863P, S241T, N395K, I136V, V1316A, G616R, G856D, I848T, I234T, and F216S [4, 6, 11, 14, 16, 18-23].

Mutations I848T and $\mathrm{L} 858 \mathrm{H}$ are located in the S4-S5 linker region of the domain II (DIIS4-S5) on the gene SCN9A. The mutated channels have shown a few differences in comparison with the wild type channel NaV1.7. Both mutations shift the voltage dependence of activation. All of the changes that have been found increase excitability of spinal sensory neurons which express NaV1.7 [7]. Rush et al. have demonstrated that the $\mathrm{L} 858 \mathrm{H}$ mutation makes the neurons of dorsal root ganglia (GDK) hyperexcitable, while it makes the sympathetic ganglia neurons hypoexcitable [23]. Yang et al. have shown that there is also a mutation I848T which is linked to IEM [1]. Cummins et al. have shown that these mutations affect the biophysical properties on these channels because both of these mutations cause a significant shift of hyperpolarization in the $\mathrm{V}_{1 / 2}$ channel activation [7]. F1449V and A853P mutations resulted in a lower threshold of triggering the action potential in the GDK and in a greater intensity of responses to stimuli compared to controls [14].

Mutation N395K, located on the S6 segment of domain I, is associated with a very serious form of IEM. The mentioned mutation is located on the gene segment which is responsible for the transcription of the locus which binds local anesthetic. Therefore, it directly affects the interaction of local anesthetic with the channel by reducing their sensitivity to the local anesthetic. This has been proved on HEK293 cells. Mutation F216S on the same gene was inserted in the HEK293 cell line by transfection, but this mutation is (unlike previous) located outside of the locus which binds local anesthetic. This has proved that this mutation, unlike N395K, affects the voltage dependent characteristics of the channel but does not affect the interaction with local anesthetics. The explanation of this phenomenon is that the channels with the mutation N395K have a disturbed inactivation and this reduces the number of channels which bind a local anesthetic (lidocaine in this case). Other scientists explain this phenomenon by the fact that this mutation impacts the direct interaction of lidocaine with the channel in inactive state [16].

The effects of S241T mutations are reducing the time to achieve the peak of action potential, slow deactivation, and an increased sensitivity of the channels to low stimuli. Serine (S) and threonine $(\mathrm{T})$ are amino acids with similar biochemical characteristics because of the presence of reactive $\mathrm{OH}$ group in the side chains. Therefore, it is expected that the protein will retain its basic function due to the substitution S241T. However, Serine on the position 241 in S4-S5 linker region is a potential place for the binding of phosphorus, which is a part of a protein kinase C molecule. Ser-241 is highly conserved residua in DI S4-S5 linker region in all of the membrane channels of vertebrates. Any change in this residue, even with a biochemically similar amino acid, causes changes in the porosity of the channel and its function. Also, the side chain of threonine is larger than the side chain of Serine and that also has the effect on the changing of the channel function [4].

Ahn et al. have identified the mutation on the SCN9A gene, which results in the substitution of Isoleucine with threonine (I234T). Cells with this mutation show a shift in the voltage dependent activation, rapid reach of the peak, slower deactivation, and an increased response to depolarization [18].

Further researches continue in the group of patients with IEM, in the group of patients with insufficient manifested symptoms of IEM, and in the group of patients with combined symptoms of IEM. In such patients the mutation 4894G > A, A1632T, on the gene for NaV1.7 has been observed [17].

\section{Paroxysmal Pain Disorder and Mutations on the SCN9A Gene}

Paroxysmal extreme pain disorder (PEPD) is a rare disorder which is characterized with the episodes of pain in the form of burning in rectal, ocular, and mandibular region associated with autoimmune manifestations in the form of skin redness. It is described that the first symptoms of disease occur in the neonatal period or in the early childhood and that they retain through their lives [24].

All of the 10 known missense mutations of the NaV1.7 on the gene SCN9A which have impact on the occurrence of PEPD cause the changes in channel closing (inactivation). Reduced inactivation of these channels causes prolonged action potential and repetitive firing. The most frequent mutation is $\mathrm{M} 1627 \mathrm{~K}[2,17,25]$.

\section{Congenital Analgesia and Mutations on SCN9A Gene}

Congenital insensitivity to pain (CIP) represents an extremely rare disorder in which a person cannot feel the pain. The genetic basis of this disorder also lies in the mutations of the SCN9A gene [2]. Some of these mutations are S459X, I767X, W897X, M899I, and M932L [10, 26-28].

Research conducted by Yuan et al. included the analysis of the sequences of the candidate genes. Two heterozygous mutations (M899I and M932L) in the gene SCN9A were found and it is considered that they are responsible for the onset of this disease. However, a recently discovered, nonsynonymous polymorphism on one nucleotide (single nucleotide polymorphism, SNP) c.3312G>T, inside of exon 16 of the gene SCN9A, results in the V1104L amino acid substitution in the human NaV1.7. This SNP has been present in those who have a decreased sensitivity to pain as well as in healthy family members. Due to the fact that this SNP is present in the genome of $6.5 \%$ of the healthy Chinese population, it is 
assumed that this mutation may be responsible for the variation in the sensitivity of pain in the general population [26].

\section{Link between the Pain Perception Disorders and Mutations on the Gene SCN9A}

Mutation A1632T was found in the patients with a combination of symptoms that characterize both disorders, IEM and PEPD. Eberhardt et al. have implemented the mutated DNA (previously extracted from lymphocytes and epithelial cells of oral mucosa) by transfection into the HEK 293 cells (human embryonic kidney 293 cells) and provoke the T/E/D/K/V substitutions of amino acids on the A1632 residue. They observed the consequential effect on the conductivity of the cell membrane. All the exons as well as the border parts between the exons and introns on the SCN9A gene were amplified by the PCR technique, using genetically specific intronic primers. Mutations A1632D/E/K/T were implemented in the splicing variants of the gene NaV1.7 and then the gene was introduced by transfection into the HEK 293 cells. After the establishment of the stable cell culture, which level of action potential is needed to cause the cell excitation was examined. Mutations T/D/E (substitution of alanine by threonine/aspartame/glutamic acid) on the position 1632 cause a significant shift of the fast inactivation, while the substitution of alanine by valine and lysine does not modify the fast inactivation. These data indicate that the size and the possible conformation changes in the protein as well as mutation of the gene may lead to the changes in the conduction of the action potential and in the perception of pain [17].

Some mutations, like A1632E, change the function of NaV1.7 channel. This is reflected in the combination of two out of three previously mentioned syndromes. This suggests that further research of the mutations on this gene could answer the question: what intensity of pain do patients actually feel in the presence of stimulation [2].

\section{Application of Research in Anesthesiology}

Studies conducted on the SCN9A gene indicate that the changes in the NaV1.7 protein structure play the key role in the occurrence of pain and suggest that the IEM, PEPD, and CIP may serve as disease models for the future research about appropriate targeted pain therapy [16]. It is evident that patients respond differently to current treatment strategies, so it is almost impossible for the clinician to assume the reaction of the individual and the specific dose to be applied. Drugs that inhibit voltage-gated sodium channels are being used in the treatment of pain, and this includes local anesthetics and Nav-blocking anticonvulsants [29]. The importance of Nav receptors in pain treatment is proved in studies [30, 31]. New drugs are being discovered, and they should be more specific, since it has been proved that gene mutations have a consequence of different level of drug-receptor binging [32].

Duan et al. investigated if the polymorphism 3312G $>\mathrm{T}$ on the gene SCN9A is responsible for the intensity of pain perception variability in the general population. The study included patients of 20-70 years of age that were subjected to pancreatectomy operations with general anesthesia $(0.05 \mathrm{mg} / \mathrm{kg}$ Midazolam, $2 \mathrm{mg} / \mathrm{kg}$ Propofol, $0.5 \mu \mathrm{g} / \mathrm{kg}$ Sufentanil, and $0.6 \mathrm{mg} / \mathrm{kg}$ Rocuronium for the induction of anesthesia, inhalation of $1 \%$ Sevoflurane, and infusion of $0.2-0.4 \mu \mathrm{g} / \mathrm{kg} / \mathrm{min}$ Remifentanil, $6-10 \mathrm{mg} / \mathrm{kg} / \mathrm{h}$ Propofol, and $0.2 \mathrm{mg} / \mathrm{kg}$ Rocuronium for the maintenance of anesthesia). $40 \mathrm{mg}$ of parecoxib sodium and $2 \mathrm{mg}$ of tropisetron hydrochloride were given intravenously fifteen minutes before incision for the alleviation of postoperative pain and prevention of postoperative nausea and vomiting. Immediately after surgery it has been enabled for patients to receive $0.5 \mu \mathrm{g} / \mathrm{mL}$ Sufentanil and $5 \mathrm{mg} / \mathrm{mL}$ Tramadol in controlled infusion. It has been statistically proved that the patients homozygous for the allele $3312 \mathrm{G}$ had a greater need for postoperative analgesia than those who were heterozygous for 3312 T allele. This research demonstrated that the reduction in the sensitivity to postoperative pain is linked to SNP SCN9A3312G $>$ T. The patients carriers of the SCN9A3312T allele had lower sensitivity to similar operative stimuli and required a lower level of analgesia than those who carried the 3312G allele [9].

A comprehensive study conducted by Reimanna et al. indicated the importance of the R1150W SNP on the SCN9A gene for the increased pain perception in the patients with osteoarthritis, patients after lumbar discectomy, amputees with phantom pain, pain-assessed people with sciatica, and patients with pancreatitis. Genotypisation proved that there exists a significant correlation between greater pain perception and A allele, which is less common in general population. The result was confirmed in a group of healthy women who were exposed to noxious stimuli [33]. This result was confirmed by Valdes et al. on the patients suffering from osteoarthritis [34]. However, Holliday et al. have denied these studies with the claims that this mutation is not connected with the greater pain perception, and this was explained by the fact that their sample was heterogenous. The authors also point out that more comprehensive and more specific studies are required in the future [35].

When it comes to the treatment of chronic pain, there are demands which clinicians can rarely follow, because the accurate target therapy based on genetic testing is needed. Theile and Cummins have showed, based on the research conducted on stably transfected human embrionic kidney cells, that the most effective therapy in the case of IEM is Lidocaine, Aspirin, Mexiletine, and Gabapentin, while, in the case of PEPD, the most effective therapy is Carbamazepine, Riluzole, Anandamide, and Lacosamide [36].

Drugs which block sodium channels, such as local anesthetics (Lidocaine), anticonvulsants (Carbamazepine, Phenytoin), and tricyclic antidepressants (Imipramine), were only partially successful in the case of IEM. Mutations V1316A, I848T, and I136V on the gene SCN9A showed a higher resistance to Lidocaine when compared to wild type channels in the experiment conducted by $\mathrm{Wu}$ et al. An alternative to Lidocaine could be Mexiletine, since it showed more efficiency than Lidocaine, especially in patients with I848T mutation $[6,37]$. It has also been shown that Mexiletine is an adequate replacement for Lidocaine in the case of L858F mutation [38]. 


\section{Conclusion}

Treatment of pain disorders has not proved to be successful in long-term application. Modern researches in pharmacogenomics suggest that further understanding of NaV1.7 channel function and pharmacology will be crucial for understanding of the pathophysiology of pain and for the development of the appropriate targeted pain treatment. Pain perception disorders are very rare; however, the findings suggest that there is a high possibility that the allelic differences in the SCN9A gene affect the pain perception in general population. Therefore, it will be possible to apply targeted therapy in everyday clinical practice, especially in intensive care units, in patients with normal pain threshold. In this way it would be possible to facilitate postoperative course, to avoid unwanted effects of pain treatment, and to reduce the number of postoperative hospital days.

\section{Conflict of Interests}

The authors declare that there is no conflict of interests regarding the publication of this paper.

\section{References}

[1] Y. Yang, Y. Wang, S. Li et al., "Mutations in SCN9A, encoding a sodium channel alpha subunit, in patients with primary erythermalgia," Journal of Medical Genetics, vol. 41, no. 3, pp. 171-174, 2004.

[2] M. Estacion, S. D. Dib-Hajj, P. J. Benke et al., "NaV1.7 gain-offunction mutations as a continuum: a1632E displays physiological changes associated with erythromelalgia and paroxysmal extreme pain disorder mutations and produces symptoms of both disorders," The Journal of Neuroscience, vol. 28, no. 43, pp. 11079-11088, 2008.

[3] C. J. Laedermann, N. Syam, M. Pertin, I. Decosterd, and H. Abriel, " $\beta 1$ - and $\beta 3$-voltage-gated sodium channel subunits modulate cell surface expression and glycosylation of Nav1.7 in HEK293 cells," Frontiers in Cellular Neuroscience, vol. 7, pp. 1-12, 2013.

[4] A. Lampert, S. D. Dib-Hajj, L. Tyrrell, and S. G. Waxman, "Size matters: erythromelalgia mutation S241T in Nav1.7 alters channel gating," The Journal of Biological Chemistry, vol. 281, no. 47, pp. 36029-36035, 2006.

[5] M. H. Meisler and J. A. Kearney, "Sodium channel mutations in epilepsy and other neurological disorders," The Journal of Clinical Investigation, vol. 115, no. 8, pp. 2010-2017, 2005.

[6] M.-T. Wu, P.-Y. Huang, C.-T. Yen, C.-C. Chen, and M.-J. Lee, "A novel SCN9A mutation responsible for primary erythromelalgia and is resistant to the treatment of sodium channel blockers," PLoS ONE, vol. 8, no. 1, Article ID e55212, 2013.

[7] T. R. Cummins, S. D. Dib-Hajj, and S. G. Waxman, "Electrophysiological properties of mutant $\mathrm{Na}_{v} 1.7$ sodium channels in a painful inherited neuropathy," The Journal of Neuroscience, vol. 24, no. 38, pp. 8232-8236, 2004.

[8] J. P. H. Drenth and S. G. Waxman, "Mutations in sodiumchannel gene SCN9A cause a spectrum of human genetic pain disorders," The Journal of Clinical Investigation, vol. 117, no. 12, pp. 3603-3609, 2007.

[9] G. Duan, G. Xiang, X. Zhang, R. Yuan, H. Zhan, and D. Qi, "A single-nucleotide polymorphism in SCN9A may decrease postoperative pain sensitivity in the general population," Anesthesiology, vol. 118, no. 2, pp. 436-442, 2013.

[10] J. J. Cox, F. Reimann, A. K. Nicholas et al., "An SCN9A channelopathy causes congenital inability to experience pain," Nature, vol. 444, no. 7121, pp. 894-898, 2006.

[11] J.-S. Choi, X. Cheng, E. Foster et al., "Alternative splicing may contribute to time-dependent manifestation of inherited erythromelalgia," Brain, vol. 133, no. 6, pp. 1823-1835, 2010.

[12] S. M. Kuhnert, W. J. Phillips, and M. D. P. Davis, "Lidocaine and mexiletine therapy for erythromelalgia," Archives of Dermatology, vol. 135, no. 12, pp. 1447-1449, 1999.

[13] M. D. Davis and P. Sandoroni, "Lidocaine patch for pain of erytromelalgia," Archives of Dermatology, vol. 138, no. 1, pp. 1719, 2002.

[14] S. D. Dib-Hajj, A. M. Rush, T. R. Cummins et al., "Gain-offunction mutation in Nav1.7 in familial erythromelalgia induces bursting of sensory neurons," Brain, vol. 128, no. 8, pp. 18471854, 2005.

[15] E. Legroux-Crespel, B. Sassolas, G. Guillet, I. Kupfer, D. Dupre, and L. Misery, "Treatment of familial erythermalgia with the association of lidocaine and mexiletine," Annales de Dermatologie et de Vénéréologie, vol. 130, no. 4, pp. 429-433, 2003.

[16] P. L. Sheets, J. O. Jackson II, S. G. Waxman, S. D. Dib-hajj, and T. R. Cummins, "A Nav 1.7 channel mutation associated with hereditary erythromelalgia contributes to neuronal hyperexcitability and displays reduced lidocaine sensitivity," Journal of Physiology, vol. 581, no. 3, pp. 1019-1031, 2007.

[17] M. Eberhardt, J. Nakajima, A. B. Klinger et al., "Inherited pain sodium channel nav1.7 A1632T mutation causes erythromelalgia due to a shift of fast inactivation," The Journal of Biological Chemistry, vol. 289, no. 4, pp. 1971-1980, 2014.

[18] H.-S. Ahn, S. D. Dib-Hajj, J. J. Cox et al., "A new Nav1.7 sodium channel mutation I234T in a child with severe pain," European Journal of Pain, vol. 14, no. 9, pp. 944-950, 2010.

[19] C. Han, A. M. Rush, S. D. Dib-Hajj et al., "Sporadic onset of erythermalgia: a gain-of-function mutation in Nav1.7," Annals of Neurology, vol. 59, no. 3, pp. 553-558, 2006.

[20] T. P. Harty, S. D. Dib-Hajj, L. Tyrrell et al., "Nav1.7 mutant A863P in erythromelalgia: effects of altered activation and steady-state inactivation on excitability of nociceptive dorsal root ganglion neurons," Journal of Neuroscience, vol. 26, no. 48, pp. 1256612575, 2006.

[21] X. Cheng, S. D. Dib-Hajj, L. Tyrrell, and S. G. Waxman, "Mutation I136V alters electrophysiological properties of the NaV1.7 channel in a family with onset of erythromelalgia in the second decade," Molecular Pain, vol. 4, article 1, 2008.

[22] J. G. J. Hoeijmakers, C. Han, I. S. J. Merkies et al., "Small nerve fibres, small hands and small feet: a new syndrome of pain, dysautonomia and acromesomelia in a kindred with a novel NaV1.7 mutation," Brain, vol. 135, no. 2, pp. 345-358, 2012.

[23] A. M. Rush, S. D. Dib-Hajj, S. Liu, T. R. Cummins, J. A. Black, and S. G. Waxman, "A single sodium channel mutation produces hyper- or hypoexcitability in different types of neurons," Proceedings of the National Academy of Sciences of the United States of America, vol. 103, no. 21, pp. 8245-8250, 2006.

[24] C. R. Fertleman, C. D. Ferrie, J. Aicardi et al., "Paroxysmal extreme pain disorder (previously familial rectal pain syndrome)," Neurology, vol. 69, no. 6, pp. 586-595, 2007.

[25] S. D. Dib-Hajj, M. Estacion, B. W. Jarecki et al., "Paroxysmal extreme pain disorder M1627K mutation in human Nav 1.7 renders DRG neurons hyperexcitable," Molecular Pain, vol. 4, article 37, 2008. 
[26] R. Yuan, X. Zhang, Q. Deng et al., "Two novel SCN9A gene heterozygous mutations may cause partial deletion of pain perception," Pain Medicine, vol. 12, no. 10, pp. 1510-1514, 2011.

[27] F. B. J. Young, "A life without pain? Hedonists take note," Clinical Genetics, vol. 73, no. 1, pp. 31-33, 2008.

[28] Y. P. Goldberg, J. Macfarlane, M. L. Macdonald et al., "Lossof-function mutations in the Nav1.7 gene underlie congenital indifference to pain in multiple human populations," Clinical Genetics, vol. 71, no. 4, pp. 311-319, 2007.

[29] J. J. Clare, "Targeting voltage-gated sodium channels for pain therapy," Expert Opinion on Investigational Drugs, vol. 19, no. 1, pp. 45-62, 2010.

[30] I. T. Strickland, J. C. Martindale, P. L. Woodhams, A. J. Reeve, I. P. Chessell, and D. S. McQueen, "Changes in the expression of NaV1.7, NaV1.8 and NaV1.9 in a distinct population of dorsal root ganglia innervating the rat knee joint in a model of chronic inflammatory joint pain," European Journal of Pain, vol. 12, no. 5, pp. 564-572, 2008.

[31] W. Wang, J. Gu, Y.-Q. Li, and Y.-X. Tao, "Are voltage-gated sodium channels on the dorsal root ganglion involved in the development of neuropathic pain?" Molecular Pain, vol. 7, article 16, 2011.

[32] J. R. Walkera, P. A. Novicka, W. H. Parsonsa et al., "Marked difference in saxitoxin and tetrodotoxin affinity for the human nociceptive voltage-gated sodium channel (Nav1.7)," Proceedings of the National Academy of Sciences of the United States of America, vol. 109, no. 44, pp. 18102-18107, 2012.

[33] F. Reimanna, J. J. Cox, I. Belfer et al., "Pain perception is altered by a nucleotide polymorphism in SCN9A," Proceedings of the National Academy of Sciences of the United States of America, vol. 107, no. 11, pp. 5148-5153, 2010.

[34] A. M. Valdes, N. K. Arden, F. L. Vaughn et al., "Role of the $\mathrm{Na}_{v} 1.7$ R1150W amino acid change in susceptibility to symptomatic knee osteoarthritis and multiple regional pain," Arthritis Care \& Research, vol. 63, no. 3, pp. 440-444, 2011.

[35] K. L. Holliday, W. Thomson, T. Neogi et al., "The nonsynonymous SNP, R1150W, in SCN9A is not associated with chronic widespread pain susceptibility," Molecular Pain, vol. 8, article 72, 2012.

[36] J. W. Theile and T. R. Cummins, "Inhibition of Nav $\beta 4$ peptidemediated resurgent sodium currents in Nav1.7 channels by carbamazepine, riluzole, and anandamide," Molecular Pharmacology, vol. 80, no. 4, pp. 724-734, 2011.

[37] D. M. Kullmann and S. G. Waxman, "Neurological channelopathies: new insights into disease mechanisms and ion channel function," Journal of Physiology, vol. 588, no. 11, pp. 1823-1827, 2010.

[38] R. Cregg, J. J. Cox, D. L. H. Bennett, J. N. Wood, and R. Werehausen, "Mexiletine as a treatment for primary erythromelalgia: normalization of biophysical properties of mutant L858F Nav1.7 sodium channels," British Journal of Pharmacology, vol. 171, no. 19, pp. 4455-4463, 2014. 


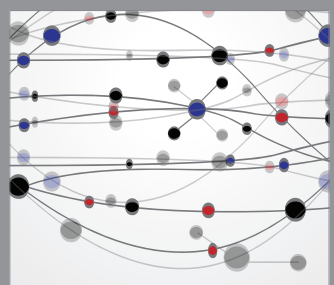

The Scientific World Journal
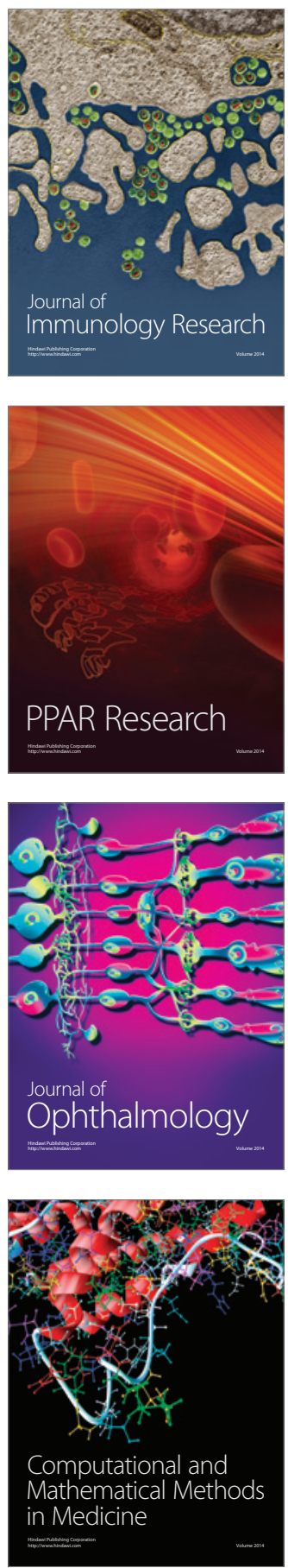

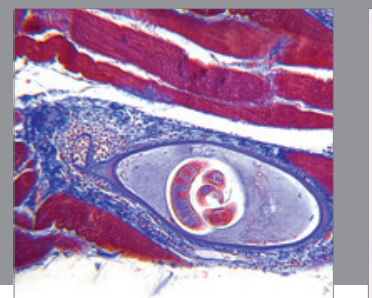

Gastroenterology

Research and Practice
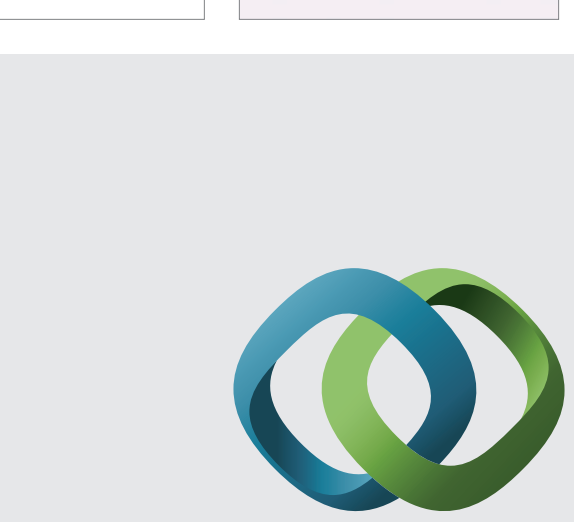

\section{Hindawi}

Submit your manuscripts at

http://www.hindawi.com
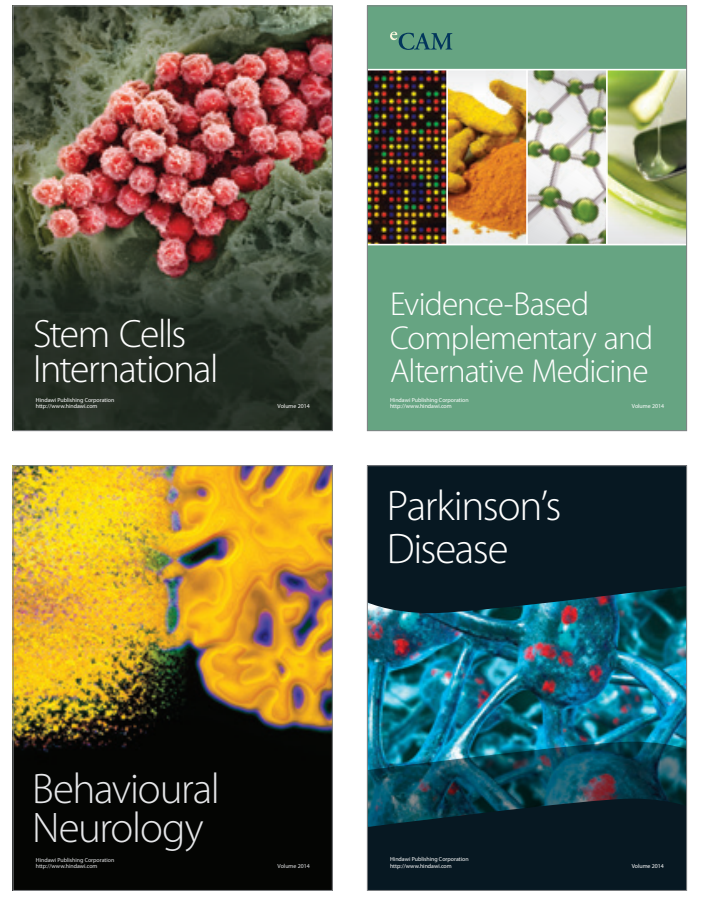
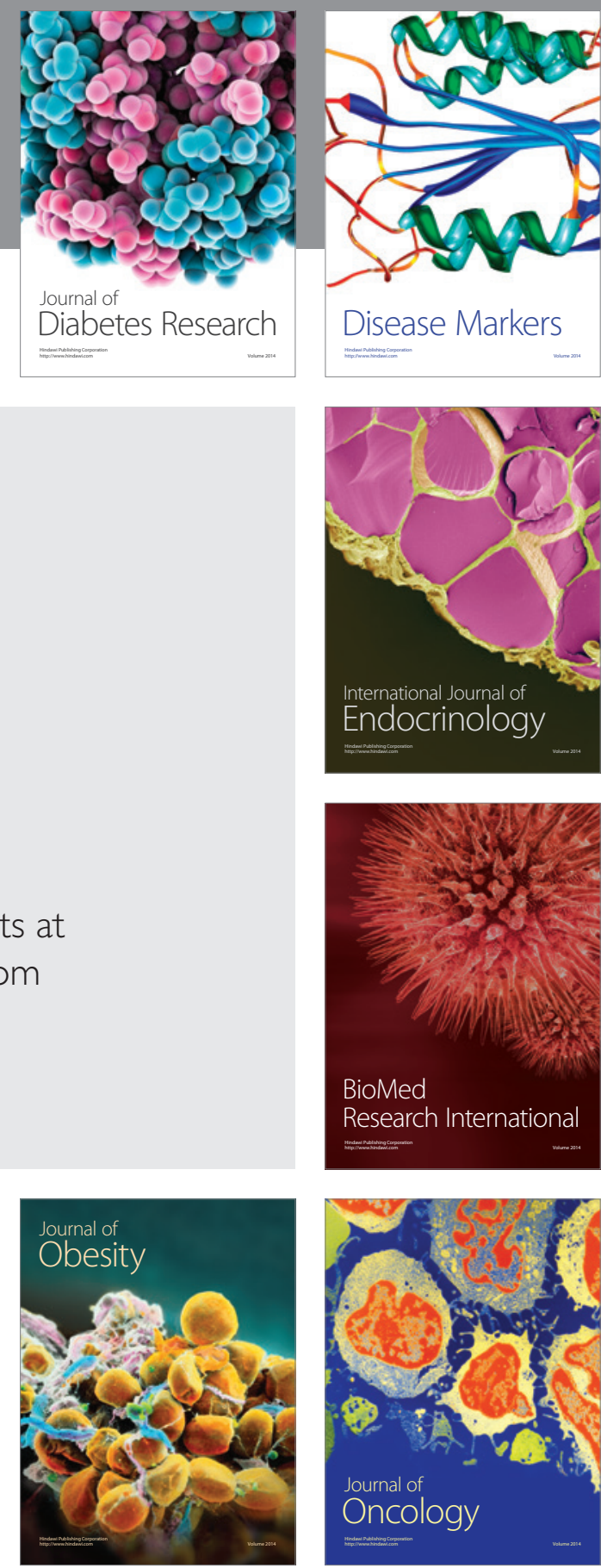

Disease Markers
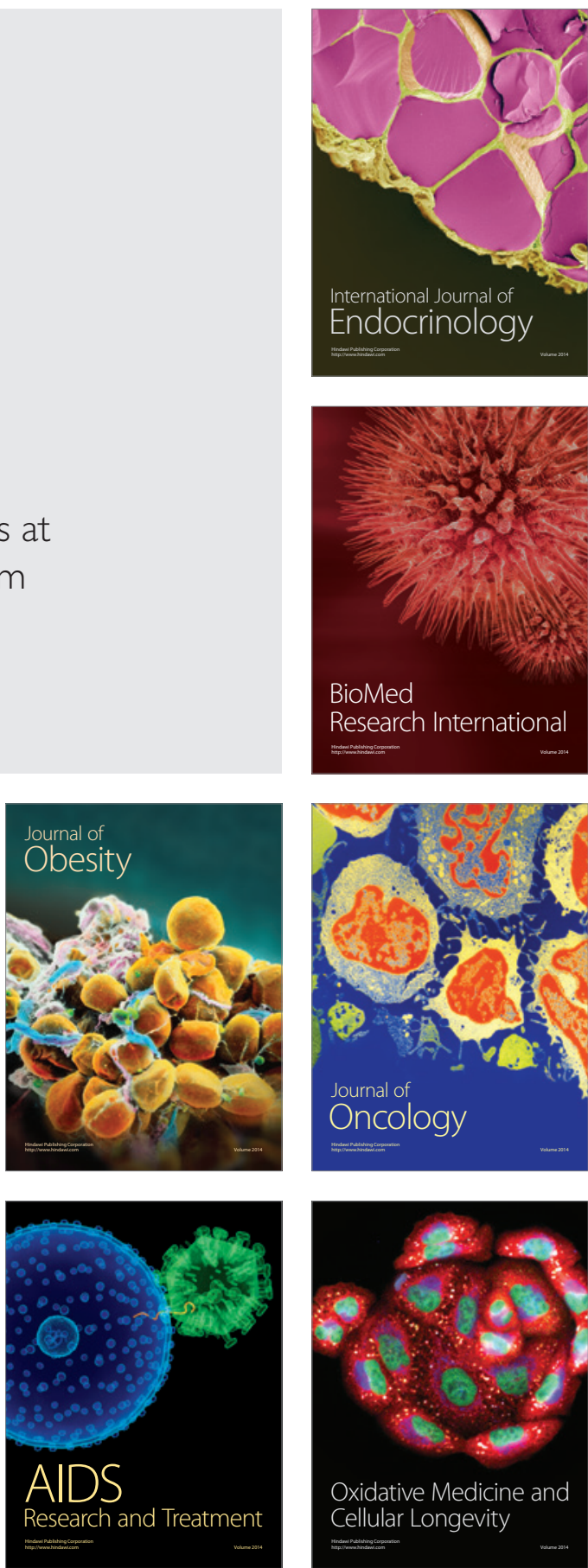